\title{
The "Non-Locality" of Entangled States Is Seeming Phenomenon
}

\author{
Marat Guryev \\ Shipilovskaya Street, Moscow, Russia \\ Email: mvguryev@mail.ru
}

How to cite this paper: Guryev, M. (2017) The "Non-Locality" of Entangled States Is Seeming Phenomenon. Journal of Applied Mathematics and Physics, 5, 1791-1796. https://doi.org/10.4236/jamp.2017.59151

Received: August 20, 2017

Accepted: September 19, 2017

Published: September 22, 2017

Copyright $\odot 2017$ by author and Scientific Research Publishing Inc. This work is licensed under the Creative Commons Attribution International License (CC BY 4.0).

http://creativecommons.org/licenses/by/4.0/

\begin{abstract}
EPR raised fundamental problems of non-locality (NL) in the case of entangled states (ES) 82 years ago. These problems were not solved until now. EPR and their followers used and would continue to use calculation methods that were available at that time. However, we can easily explain this observable NL as a trivial result of conservation laws (CL) within modern quantum mechanics (MQM). But application of CL requires materialistic descriptions of reality in a micro world in contrast to so-called quantum measurement theory (QMT), which was created mainly in the times of EPR and is widely accepted until now. We have to use a materialistic description, just as many physicists who actually work with high precision do by default. In this article, practical examples are given for real, precise measurements of wave functions of molecules and crystals, which, of course, were not known to EPR and were not noticed by their followers. We should acknowledge that QMT is merely an unneeded complication of simple relations of MQM. NL is the seeming result of these complications.
\end{abstract}

\section{Keywords}

Non-Locality, Entangled States, Quantum Measurement Theory, EPR, Electron Diffraction, Neutron Diffraction, $\gamma-$ Ray Diffraction, Measurement of Wave Function

\section{Introduction}

Quantum entanglement (QE) is a field of very active study. It is well-described in extensive physical literature (see, e.g., the large review article [1]). Einstein, Podolsky, and Rosen (EPR) raised fundamental problems of non-locality (NL) of entangled states (ES) 82 years ago. We will not consider problems connected with hidden variables because these problems are now regarded as solved. Prob- 
lems of NL were widely discussed. Some authors came up to talks even about counterfactual (unfeasible) objects, see e.g. [2]. This approach resulted in insignificant useful results [3].

It is necessary to note that EPR could not use for consideration of QE practical calculation methods of modern quantum mechanics (MQM), which were unknown at that time.

We can express as follows the main apparent properties of QE, which EPR accepted with certainty as a non-locality within QM. The experiment on particle 1 influences the result of the experiment on particle 2, although these particles in ES are widely separated.

A contemporary physicist, if he is a specialist in precise work, seeing such reasoning, will say at once that we should use conservation laws (CL) in this case. Observable, seeming long-range interactions of spins for atoms at macroscopic distances are mere results of CL, which are described in serious textbooks on quantum mechanics, textbooks on quantum collision theory (QCT) (see, e.g., [4] [5] [6] [7]), and textbooks on quantum electrodynamics (QED) [8] [9].

The problem is that, in practice, we use by default real values of corresponding vectors in CL but not results of applications to them of quantum measurement theory (QMT) as EPR has done. Work with reality implies that we are materialists without complications introduced by QMT.

It is worth noting that there was not a trial to give up application of QMT. Actually, it was a deadlock. We will look for the way to break the deadlock.

The crucial role of QMT in problems of ES with NL was apparently, never carefully highlighted. The fact of the matter is that the main statement of QMT, which was actually used in consideration of problems of ES, was in use by default. It is possible to say that serious details of QMT are actually "unspeakable" in considerations of $\mathrm{QE}$ and non-locality.

I draw the reader's attention to the fact that QCT, as well as corresponding experiments within large distances, is impossible without using CL. This assertion relates also to high-energy physics, which represents the ultra-relativistic part of QCT. Non-relativistic QCT with CL is widely in use for description of different interactions between particles, atoms, and molecules [10]. For example, one can look into the corresponding section in any modern issue of the journals Physical Review A or Journal of Chemical Physics. Thus, we can acknowledge that it is widely accepted by default that QCT and all its details are real objects with real properties. Therefore, these objects actually do not need some macroscopic measurement apparatus to exist and especially to impact.

We should acknowledge that actually no specialists in precise physics use QMT. Only specialists in QMT work with it. We will try to show that sophisticated reasoning of QMT is not needed if we work within MQM.

The goal of this article is to delete the reasons that have resulted in EPR's hypothesis of non-locality in QM. The rest of the article is organized as follows. In Section 2, quantum measurement problems of QMT are considered in detail 
from the point of view of modern physics. Section 3 is devoted to real measurements of wave functions of molecules; we consider these measurements an example of correct work with reality within MQM. Section 4 represents a short summary of the main reasons for confusion, which has created the seeming non-locality.

\section{Measurements in the Quantum World from the Point of View of Modern Physics}

QMT is described in numerous papers; see [11]. The main generally accepted statement of QMT, which we will further consider, can be written as follows: measured quantum states and their properties do not exist as reality before measurement [4] [7]. This controversial hypothesis is in obligatory use in QMT and, correspondingly, in descriptions of QE, for the last 82 years. It is the right time to try to look at foundations of QMT in a new way.

It is worth noting that there were several serious attempts made to make old QMT better. These trials have turned out to be not successful [12] [13] [14].

Specialists on QMT generally assume by default that their approach to measurement is the only feasible one. Thus, they assume that QMT is by default generally accepted in use for theoretical description of all the experiments in micro world. Of course, we apply QMT for measurements in Stern-Gerlach and similar experiments. But now we can measure not only the values of spins of atoms in these experiments.

We widely use eigenstates as theoretical results of MQM, especially we use precise results of QED and spectroscopy for measurement of atomic eigenstates (see, e.g., [8]). It is obvious that measurement methods of modern spectroscopy do not need direct influence of macroscopic apparatus on a light source. We also know how to measure collision cross-sections [10], various transition probabilities [4], and even wave functions of molecules [15]. We will consider the last in more detail in Section 3.

We really use all these results in applied physics, but it turns out that these real measurements have no physical meaning from the point of view of monopolistic QMT. This implies that we should get rid of the monopoly of QMT. It has turned out that we have to introduce the other definition of reality in the quantum world. This issue is not simple.

Let us note that practical applications of alternative between materialism and all other philosophical doctrines should consider not just philosophical problems but also practical decisions in day-to-day work. We directly apply such decisions in everyday practice of precise modern physics for comparison of calculated precise results with results of experiments. In every such comparison, we by default imply that the quantum world is real.

We will use following definition of reality: objects of the quantum world and their properties are real if they exist out of us and independently of us, and if results of theoretical calculations coincide well with results of experiments. The 
thoughts of professor Gisin [16] were used in the derivation of this definition.

I draw the reader's attention that just this definition is by default in practice of exact MQM and exact corresponding experiments. The physicist's task is to describe in theory just this reality as precisely as we can but not obtrude our opinion and interpretation upon nature (I remind the reader of Occam's razor). We should not philosophize in general, remembering classical Lagrange mechanics. Instead, we should study and search for calculation methods for more exact descriptions of reality that represent different precise experimental data.

Below, we will give some additional arguments in favor of this definition by known examples from modern physics.

\section{A Practical Modern Method for the Measurement of Wave Functions of Molecules}

Now we move to consideration of just practical open using of a materialistic approach for direct measurement of the modulus of wave functions of molecules [15]. Measurements of relative phases in principle are known as nice interference experiments with atoms (see, e.g., [17]), and we will not consider them.

The practical modern method of wave function measurement is called gas-phase electron diffraction. This measurement method is based on first-order approximation of the perturbation theory of QCT for elastic scattering of fast electrons by molecules. In this case, the directly observable quantity is the usual differential cross-section in atomic units and usual notations:

$\mathrm{d} \sigma=\frac{1}{k^{2}}|f(\mathbf{q})|^{2} q \mathrm{~d} q \mathrm{~d} \varphi$, where $\mathbf{q}=\mathbf{k}_{1}-\mathbf{k}_{2}$ and $\mathbf{k}_{1}$ and $\mathbf{k}_{2}$ are initial and final vectors of moment of incoming electron, herewith $k_{1}=k_{2} \equiv k$.

The scattering amplitude for a molecule that contains $N$ electrons is

$$
\begin{aligned}
f(\mathbf{q})= & \frac{1}{2 \pi} \int \exp (i \mathbf{q r})\left(\sum_{n} \exp \left(i \mathbf{q} \mathbf{r}_{1}\right)+\ldots \exp \left(i \mathbf{q r} \mathbf{r}_{n}\right)+\ldots \exp \left(i \mathbf{q} \mathbf{r}_{N}\right)\right) \times \\
& \int\left|\psi\left(\mathbf{r}_{1} \ldots \mathbf{r}_{n} \ldots \mathbf{r}_{N}\right)\right|^{2} \mathrm{~d}\left(\mathbf{r}_{1} \ldots \mathbf{r}_{n} . . \mathbf{r}_{N}\right) \frac{\mathrm{d} \mathbf{r}}{r}
\end{aligned}
$$

The corresponding calculation method for molecules is described in detail in [15].

There are actually many different measuring methods in first-order approximation within MQM. I remind the reader that there are different crystallographic methods in which contemporary physicists study scattering of X-rays, scattering of fast electrons, and scattering of neutrons by crystals. Scattering of $\mathrm{X}$-rays is the main method of MQM to measure with high precision the structures of crystals.

In this case, we can consider a crystal as a single molecule, and all these methods can be considered similar in principle to the above method of gas-phase electron diffraction. There are also methods of measurement of some quantities related to wave functions. For example, we may offer a direct nondestructive method for measurement of speed of electrons with small magnetic fields and 
direct measurement of radiation [18]. It is possible to carry out by scattering the direct measurement of spin orientation of an atom or electron. The corresponding theory is given in textbooks on QED (see, e.g., [9]). Finally, we can run measurement of spins of atoms in Stern-Gerlach experiments using the scattering method. Thus, QMT has no monopoly even in this case.

Apparently, every case of interaction in a continuum that can be described precisely enough by known methods of QCT should be considered as a base of possible new measurement methods. Of course, we assume that in a gas phase, we must have very many identical objects for carrying out our measurement, as well as in QMT. That is, we must use this part of old QMT.

\section{Conclusions}

The main reason for the confusion, which occurred with non-locality and ES, is the application of out-of-date QMT, which claims: measured quantum states and their properties do not exist as reality before measurement. We propose simple solution of this confusion. The main step in this direction is the acknowledgement of reality within MQM. Firstly, we should acknowledge the numerous experimental results, obtained by gas-phase electron diffraction methods [15] for measurement of wave functions of molecules and by different crystallographic methods for measurement of wave functions of crystals. These results represent obvious experimental validation of a materialistic approach to the description of MQM in principle. Secondly, we should understand that we actually acknowledge reality of MQM comparing results of experiment with results of calculations. We can see that in such approach, there are no non-localities. Thus, we must manage within materialistic methods without the EPR hypothesis of non-locality and QMT.

\section{Acknowledgements}

The author thanks reviewer for his work.

\section{References}

[1] Horodecki, R., Horodecki, P., Horodecki, M. and Horodecki, K. (2009) Quantum Entanglement. Reviews of Modern Physics, 81, 865. https://doi.org/10.1103/RevModPhys.81.865

[2] Arvidsson-Shukur, D.R.M. and Barnes, C.H.W. (2016) Quantum Counterfactual Communication without a Weak Trace. Physical Review A, 94, Article ID: 062303. https://doi.org/10.1103/PhysRevA.94.062303

[3] Stefan, W. (2015) Non-Locality without Counterfactual Reasoning. Physical Review $A$, 92, Article ID: 052102. https://doi.org/10.1103/PhysRevA.92.052102

[4] Schwabl, F. (2007) Quantum Mechanics. 4th Edition, Springer, Berlin Heidelberg New York.

[5] Basdevant, J.L. and Dalibard, J. (2002) Quantum Mechanics. Springer, Berlin.

[6] Gasioroviczs, S. (2003) Quantum Physics. 3rd Edition, John Wiley and Sons Ltd., New York 
[7] Landau, L.D. and Lifshitz, E.M. (1989) Quantum Mechanics: Nonrelativistic Theory. Translated Fourth Russian Edition, Pergamon Press, Oxford.

[8] Grant, I.P. (2007) Relativistic Quantum Theory of Atoms and Molecules. Springer, Science-Business Media, New York. https://doi.org/10.1007/978-0-387-35069-1

[9] Berestetskii, V.B., Lifshitz, E.M. and Pitaevskii, L.P. (1982) Quantum Electrodynamics. 2nd Edition, Elsevier Ltd., Amsterdam.

[10] Joachain, C.J. (1983) Quantum Collision Theory. 3rd Edition, North-Holland Physics Publishing, Amsterdam.

[11] Wheeler, J.A. and Zurek, W.H. (2014) Quantum Theory and Measurement. Princeton Legacy Library, Princeton.

[12] Dressel, J., Malik, M., Miatto, F., Jordan, A. and Boyd, R. (2013) Understanding Quantum Weak Values: Basics and Applications.

[13] Svensson, B.E.Y. (2014) On the Interpretation of Quantum Mechanical Weak Values. Physica Scripta, 2014, Article ID: 014025. https://doi.org/10.1088/0031-8949/2014/T163/014025

[14] Knee, G.C., Combes, J., Ferrie, C. and Gauger, E.M. (2014) Weak Value Amplification: State of Play.

[15] Hargittai, I. and Hargittai, M. (1988) Stereochemical Applications of Gas-Phase Electron Diffraction, Part A, Electron Diffraction Technique. Wiley, Meinheim.

[16] Gisin, N. (2014) A Possible Definition of a Realistic Physics Theory.

[17] Schaff, J.F., Langen, T. and Schmiedmayer, J. (2014) Interferometry with Atoms. La Rivista del Nuovo Cimento, 37, 509.

[18] Asner, D.M., Bradley, R.F., et al. (2015) Single Electron Detection and Spectroscopy via Relativistic Cyclotron Radiation. Physical Review Letters, 114, Article ID: 162501. https://doi.org/10.1103/PhysRevLett.114.162501

\section{Submit or recommend next manuscript to SCIRP and we will provide best} service for you:

Accepting pre-submission inquiries through Email, Facebook, LinkedIn, Twitter, etc. A wide selection of journals (inclusive of 9 subjects, more than 200 journals)

Providing 24-hour high-quality service

User-friendly online submission system

Fair and swift peer-review system

Efficient typesetting and proofreading procedure

Display of the result of downloads and visits, as well as the number of cited articles

Maximum dissemination of your research work

Submit your manuscript at: http://papersubmission.scirp.org/

Or contact jamp@scirp.org 